\title{
Inherent Stability of Multibody Systems with Variable-Stiffness Springs via Absolute Stability Theory
}

\author{
Tianyang Hua $(\mathbb{D})$ and Yinlong Hu \\ College of Energy and Electrical Engineering, Hohai University, Nanjing 211100, China \\ Correspondence should be addressed to Yinlong Hu; yinlonghu@outlook.com
}

Received 19 August 2021; Revised 19 October 2021; Accepted 29 October 2021; Published 11 November 2021

Academic Editor: Carlos Aguilar-Ibanez

Copyright (c) 2021 Tianyang Hua and Yinlong Hu. This is an open access article distributed under the Creative Commons Attribution License, which permits unrestricted use, distribution, and reproduction in any medium, provided the original work is properly cited.

In this paper, the inherent stability problem for multibody systems with variable-stiffness springs (VSSs) is studied. Since multibody systems with VSSs may consume energy during the variation of stiffness, the inherent stability is not always ensured. The motivation of this paper is to present sufficient conditions that ensure the inherent stability of multibody systems with VSSs. The absolute stability theory is adopted, and N-degree-of-freedom (DOF) systems with VSSs are formulated as a Lur'e form. Furthermore, based on the circle criterion, sufficient conditions for the inherent stability of the systems are obtained. In order to verify these conditions, both frequency-domain and time-domain numerical simulations are conducted for several typical lowDOF systems.

\section{Introduction}

Vibration control can generally be classified into passive control, active control, and semiactive control. Semiactive control integrates the advantages of both excellent control performance of active control and high reliability of passive control, which can be realized by online adjusting the characteristic parameters (such as stiffness, damping, etc.) of originally passive devices [1]. Since semiactive control devices are obtained based on their passive counterparts, it is expected to inherit the stability of passive control, which means that even though failures and improper control actions of the parameter adjustments may occur, the semiactive control system can remain stable. Such kind of property is known as the inherent stability of semiactive control systems [2]. However, it has been demonstrated that inherent stability of semiactive control systems only conditionally holds for some special semiactive devices [2].

Variable-stiffness spring (VSS) is a commonly used semiactive control device because of its effectiveness in modifying the resonance frequencies of vibration control systems [3]. In [4], the VSS-based system is applied in seismic building control, where VSS is aimed at establishing a nonresonant state against earthquake excitation. Up to now, different realizations for VSS have been proposed. For example, a resettable VSS (RVSS) is proposed to enhance the performance of a tuned mass damper system [5]. In [6], a VSS is developed by integrating the magnetorheological fluid into a damper. Based on the force-current analogy between mechanical systems and electrical circuits, a novel electrical VSS (EVSS) is proposed to reduce the suspension deflection of a seat suspension [7]. Moreover, the performance benefits of VSSs in robotic applications have been widely demonstrated $[8,9]$.

A graphical representation on the relationship among vibration control, passivity, and inherent stability is shown in Figure 1. Note that the terminologies "passive" (seen in Definition 4) and "active" are mutually exclusive with clear boundary in definition according to whether energy can be internally generated. However, whether semiactive systems are "passive" or "active" involves some abuse of notation [2]. This means that semiactive control can be further classified into "active" semiactive control and "passive" semiactive control. For example, in [2], it is demonstrated that certain semiactive devices including nonnegative variable-damping dampers, resettable-stiffness springs, and resettable- 


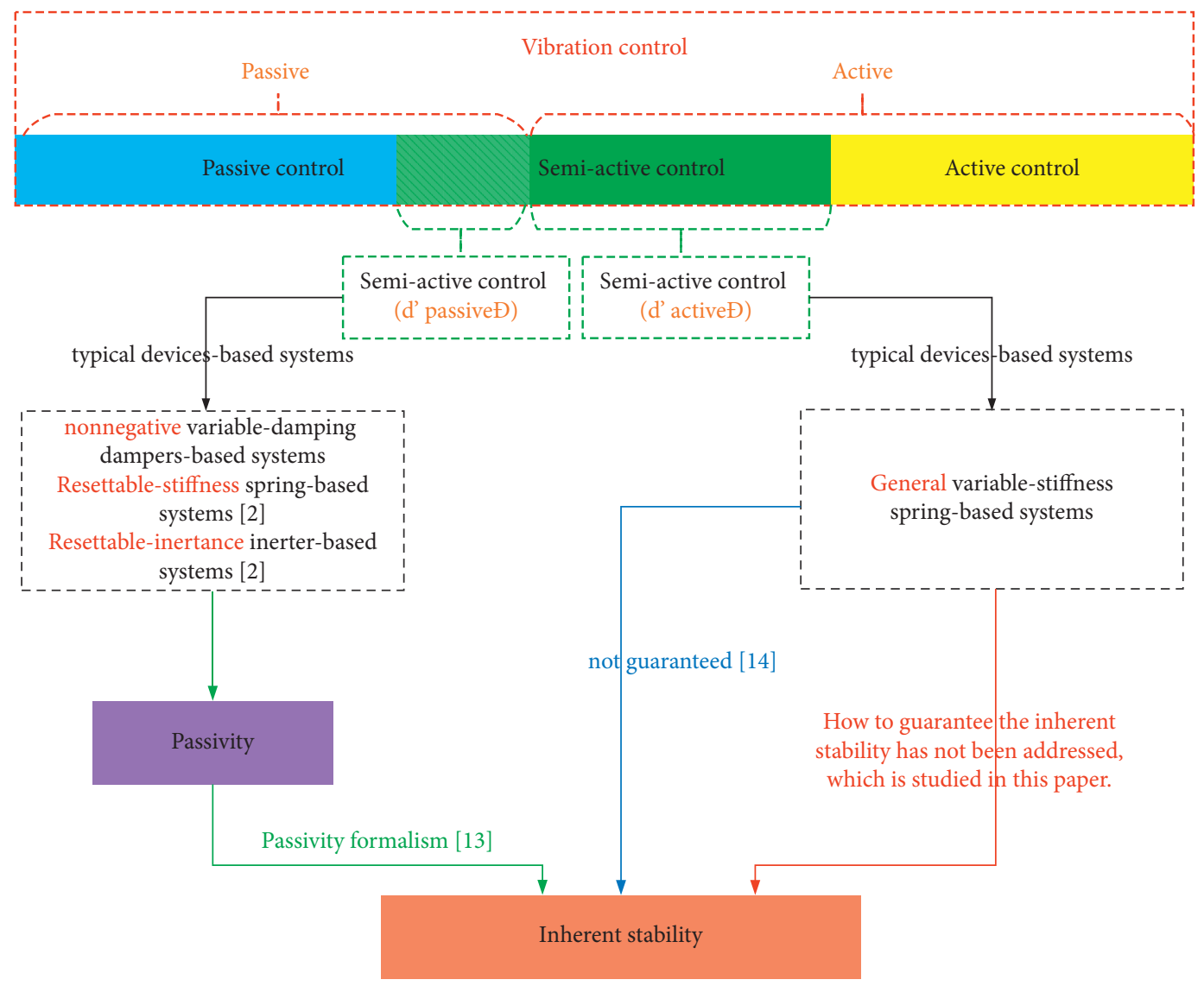

FIgURE 1: The relationship among passivity, stability, and vibration control.

inertance inerters are "passive"; in [10-12], the VSSs are actually "active" due to the less energy required for adjusting the parameters. For "passive" semiactive control systems, inherent stability can be guaranteed due to the passivity formalism [13], as shown in the green lines in Figure 1. For "active" semiactive control systems, inherent stability can not be guaranteed. In [14], it is proved that instabilities can be induced by VSSs. However, for "active" VSS-based semiactive control systems, how to ensure inherent stability has not yet been studied, which is the main concern of this paper, as shown in the red line in Figure 1. Since the stiffness influences the potential energy of a system, a certain amount of energy is generally required to adjust the stiffness. This means that "active" VSSs are more common than the "passive" ones. Therefore, it is of great theoretical and practical significance to investigate the inherent stability problem of VSSs-based systems.

The VSS-based systems are typical nonlinear control systems, where the nonlinearities are induced by the variable stiffness within a sector reflecting the bounds of VSSs. For nonlinear mechanical systems, several control strategies have been proposed to ensure stability and performance, such as sliding mode backstepping [15], adaptive finite-time control $[16,17]$, etc. In [16], the problem of finite-time faulttolerant consensus protocols for a class of uncertain multiple mechanical systems is investigated, and the proposed scheme can guarantee that the position errors and the velocity errors between any two mechanical systems converge to a small neighbourhood of zero in finite time. In [17], an adaptive fuzzy finite-time control scheme is proposed to guarantee the stability of a class of nonlinear systems with unknown nonlinearities. Note that, for the aforementioned papers, stability of the close-loop system is achieved. However, the inherent stability studied in this paper implies the stability for a group of control laws within certain sectors, instead of a special control law in the aforementioned papers. Therefore, in this paper, the inherent stability problem for the multibody systems with VSSs is studied via the absolute stability theory, and the general multibody system with VSSs is transformed into a Lur'e-type system described as a linear passive system with feedback-connected nonlinearities. The most celebrated methods to analyze the absolute stability of a Lur'e-type systems are circle criterion (CC) and Popov criterion (PC). CC can deal with more diverse nonlinearities including time-varying ones than PC [18]. Since the stiffness of VSSs is time-varying in general, CC is adopted to verify the absolute stability of the multibody systems with VSSs. Furthermore, for several typical low-DOF systems, sufficient conditions imposed on the upper and lower bounds of VSSs are derived and verified via numerical examples in both frequency domain and time domain. 
The main contributions of this paper are as follows:

(1) The inherent stability problem for the multibody systems with "active" VSSs is investigated, which has not been studied before but with great theoretical and practical significance. Sufficient conditions for the inherent stability of the general multibody systems with "active" VSSs are derived and analyzed via the absolute stability theory.

(2) The inherent stability of several typical low-DOF systems with "active" VSSs is analyzed. Moreover, extensive numerical examples based on low-DOF systems are conducted to show the effectiveness of the results of this paper.

The structure of this paper is organized as follows. Definitions and lemmas used in this paper are summarized in Section 2. In Section 3, the general multibody systems with VSSs are modeled as Lur'e-type systems, and the absolute stability of these systems is demonstrated. Numerical simulation of the absolute stability of several low-DOF systems is given in Section 4. Conclusions are drawn in Section 5 .

\section{Definitions and Lemmas}

In this section, some definitions and lemmas related to the absolute analysis of the multibody systems with VSSs are given.

Definition 1 (see [18]). The definitions of the sector terminology in both the scalar and multivariable cases are as follows:

(i) Consider a scalar function $\Psi(t, y)$ with $\Psi(t, 0) \equiv 0$, $t \geq 0$; it is said to belong to the sector $\left[K_{1}, K_{2}\right]$ if

$$
K_{1} y^{2} \leq \Psi(t, y) y \leq K_{2} y^{2}, \quad \forall t \geq 0
$$

where $K_{1}, K_{2} \in R$ and $K_{1}<K_{2}$.

(ii) Consider a specific multivariable function $\Psi(t, y)$ with $\Psi(t, 0) \equiv 0, t \geq 0$, which can be decoupled to $\Psi(t, y)=\left[\Psi_{1}\left(t, y_{1}\right), \Psi_{2}\left(t, y_{2}\right), \ldots, \Psi_{p}\left(t, y_{p}\right)\right]^{T}$; it is said to belong to the sector $\left[\mathbf{K}_{1}, \mathbf{K}_{2}\right]$ if

$$
\left[\boldsymbol{\Psi}(t, \mathbf{y})-\mathbf{K}_{1} \mathbf{y}\right]^{T}\left[\boldsymbol{\Psi}(t, \mathbf{y})-\mathbf{K}_{2} \mathbf{y}\right] \leq 0
$$

where $\quad \mathbf{K}_{1}=\operatorname{diag}\left\{\alpha_{1}, \alpha_{2}, \ldots, \alpha_{p}\right\}, \quad \mathbf{K}_{2}=\operatorname{diag}\left\{\beta_{1}\right.$, $\left.\beta_{2}, \ldots, \beta_{p}\right\}$, and $\beta_{i}>\alpha_{i}$.

Definition 2 (see [19]). Consider the Lur'e system

$$
\left\{\begin{array}{l}
\dot{\mathbf{x}}=\mathbf{A x}+\mathbf{B u}, \\
\mathbf{y}=\mathbf{C x} \\
\mathbf{u}=-\boldsymbol{\Psi}(t, \mathbf{y})
\end{array}\right.
$$

where $(\mathbf{A}, \mathbf{B})$ is controllable, $(\mathbf{A}, \mathbf{C})$ is observable, and $\Psi(t, \mathbf{y})$ is a memoryless, possibly time-varying, nonlinearity, which is piecewise continuous in $t$ and locally Lipschitz in $\mathbf{y}$. In addition, $\Psi(t, \mathbf{y})$ satisfies a sector condition per Definition
1. The system is absolutely stable if the origin is globally uniformly asymptotically stable for any nonlinearity in the given sector. It is absolutely stable with a finite domain if the origin is uniformly asymptotically stable.

Definition 3 (see [18]). Denote a disk $D\left(K_{1}, K_{2}\right)$ for $\Psi(t, y) \in\left[K_{1}, K_{2}\right]$ where $K_{1}$ and $K_{2}$ are constant, as the closed disk in the complex plane centred at $-\left(K_{1}+K_{2}\right) / 2 K_{1} K_{2}+j 0$ and with radius $\left(K_{2}-K_{1}\right) /$ $2\left|K_{1} K_{2}\right|$.

Definition 4 (see [2]). A system whose input is $\mathbf{u}_{s}$ and output is $\mathbf{y}_{s}$, for which it is defined a lower bounded (Lyapunov-like) storage function $V(t)$, is said to be "passive" if it satisfies the following equation:

$$
\dot{V}(t)=\mathbf{y}_{s}^{T} \mathbf{u}_{s}-g(t),
$$

where $g(t) \geq 0, \forall t \in[0, T]$.

Lemma 1 (see [19]). The system described in Definition 2 is absolutely stable if

(i) $\Psi \in\left[\mathbf{K}_{1}, \infty\right]$ and $\mathbf{G}(s)\left[\mathbf{I}+\mathbf{K}_{1} \mathbf{G}(s)\right]^{-1}$ is strictly positive real

(ii) $\Psi \in\left[\mathbf{K}_{1}, \mathbf{K}_{2}\right]$, with $\mathbf{K}=\mathbf{K}_{2}-\mathbf{K}_{1}=\mathbf{K}^{T}>0$, and $[\mathbf{I}+$ $\left.\mathbf{K}_{2} \mathbf{G}(s)\right]\left[\mathbf{I}+\mathbf{K}_{1} \mathbf{G}(s)\right]^{-1}$ is strictly positive real

Lemma 2 (see [19]). Consider a scalar system of equation (3), where $(\mathbf{A}, \mathbf{B}, \mathbf{C}, \mathbf{D})$ is a minimal realization of $G(s)$ and $\Psi \in\left[K_{1}, K_{2}\right]$. Then, the system is absolutely stable if one of the following conditions is satisfied, as appropriate:

(i) If $0<K_{1}<K_{2}$, the Nyquist plot of $G(j \omega)$ does not enter the disk $D\left(K_{1}, K_{2}\right)$ and encircles it $m$ times in the counterclockwise direction, where $m$ is the number of poles of $G(s)$ with positive real parts

(ii) If $0=K_{1}<K_{2}, G(s)$ is Hurwitz, and the Nyquist plot of $G(j \omega)$ lies to the right of the vertical line defined by $\operatorname{Re}(s)=-1 / K_{2}$

(iii) If $K_{1}<0<K_{2}, G(s)$ is Hurwitz, and the Nyquist plot of $G(j \omega)$ lies in the interior of the disk $D\left(K_{1}, K_{2}\right)$

If the sector condition is satisfied only on an interval $[a, b]$, the foregoing conditions ensure that the system is absolutely stable with a finite domain.

Lemma 3 (see [20]). The transfer matrix $\mathbf{G}(s)$ is a strictly positive real matrix if $\mathbf{G}(s-\varepsilon)$ is a positive real matrix for some $\varepsilon>0$.

Lemma 4 (see [21]). Let $\mathbf{G}$ be a real $m \times m$ matrix-valued rational function of the form

$$
\mathbf{G}(s)=\mathbf{C}(s \mathbf{I}-\mathbf{A})^{-1} \mathbf{B}+\mathbf{D},
$$

if the linear matrix inequalities (LMIs) 


$$
\left\{\left[\begin{array}{cc}
\mathbf{A}^{T} \mathbf{X}+\mathbf{X A} & \mathbf{X B}-\mathbf{C} \\
\mathbf{B}^{T} \mathbf{X}-\mathbf{C}^{T} & -\mathbf{D}-\mathbf{D}^{T}
\end{array}\right] \leq 0, \quad \mathbf{X} \geq 0,\right.
$$

have a solution $\mathbf{X}$ of the LMIs.

Lemma 5 (see [20]). Consider the $m \times m$ rational matrix $\mathbf{G}(s)=\mathbf{C}(s \mathbf{I}-\mathbf{A})^{-1} \mathbf{B}+\mathbf{D}$. Then $\mathbf{G}(s)$ is a strictly positive real matrix if and only if

(i) All elements of $\mathbf{G}(s)$ are analytic in $\operatorname{Re}(s) \geq 0$

(ii) $\mathbf{G}(j \omega)+\mathbf{G}^{T}(-j \omega)>0, \forall \omega \in R$

(iii) $\lim _{\omega \longrightarrow \infty} \omega^{2}\left[\mathbf{G}(j \omega)+\mathbf{G}^{T}(-j \omega)\right]>0$, if $\operatorname{det}[\mathbf{D}+$ $\left.\mathbf{D}^{T}\right]=0$ or $\lim _{\omega \longrightarrow \infty}\left[\mathbf{G}(j \omega)+\mathbf{G}^{T}(-j \omega)\right]>0$, if det

Lemma 6 (see [22]). Consider the $m \times m$ rational matrix $\mathbf{G}(s)=\mathbf{C}(s \mathbf{I}-\mathbf{A})^{-1} \mathbf{B}+\mathbf{D}$. If all the eigenvalues of $\mathbf{A}$ have negative real part, then there is a $\beta>0$ such that $\mathbf{G}$ is analytic in $\operatorname{Re}(s)>-\beta$.

\section{Absolute Stability Analysis of $\mathbf{N}$-DOF Multibody Systems with VSSs}

Consider a $N$-DOF multibody system with a dynamic equation as follows:

$$
\mathbf{M}_{p} \ddot{\mathbf{q}}(t)+\mathbf{C}_{p} \dot{\mathbf{q}}(t)+\mathbf{K}_{p} \mathbf{q}(t)=\mathbf{F}(t),
$$

where $\mathbf{q}(t) \in \mathbf{R}^{N \times 1}$ is the generalized coordinate vector of each DOF and $\mathbf{M}_{p}, \mathbf{C}_{p}$, and $\mathbf{K}_{p} \in \mathbf{R}^{N \times N}$ are the mass, damping, and stiffness matrices of the $N$-DOF multibody system, respectively. $\mathbf{F}(t) \in \mathbf{R}^{N \times 1}$ is the force applied by VSSs and is imposed on the $N$-DOF multibody system. Note that equation (7) represents a general description of multibody systems, which can be used to model vehicles, bridges, buildings, etc. The main problem of this paper is as follows.

Problem 1. Consider a N-DOF system with $n_{k}$ VSSs where the stiffness can be adjusted online within certain ranges. Determine the ranges for the stiffness of VSSs where the inherent stability can always be guaranteed.

Since it has been proved in [14] that instability can be induced by "active" VSSs, Problem 1 is intended to propose sufficient conditions for the "active" VSSs to guarantee the inherent stability on condition that the stiffness of VSSs is adjusted within certain ranges. In practice, the stiffness of VSSs is always bounded due to the physical realizations. For example, for variable-stiffness leaf springs [23], the stiffness is limited by the geometry; for a variable stiffness mechanism [24], the stiffness is limited by the rotation amount of the springs, etc. Therefore, apart from the theoretical interest of studying Problem 1, the ranges obtained from Problem 1 can provide practical guidance for the selection and manufacture of VSSs.

Assume that there are $n_{k}$ VSSs to be applied to the multibody system, where $n_{k} \leq n(n+1) / 2$ (since VSS has two mechanical terminals, there are at most $n(n+1) / 2$ VSSs that can be applied in a $N$-DOF system). The locations for connecting VSSs are numbered as $0,1,2, \ldots, n$, where location 0 is the inertial frame (mechanical ground) and locations $1,2, \ldots, n$ correspond to the $N$-DOFs, respectively. For a VSS $k_{l}, l=\left[1, \ldots, n_{k}\right]$, its two terminals are connected to the locations $i_{l}$ and $j_{l}$, where $i_{l}$ and $j_{l}$ are some integers between $[0, \ldots, n]$ and $j_{l}>i_{l}$.

Proposition 1. If $i_{l} \neq 0, \mathbf{e}_{i_{l}, j_{l}}$ is a column vector with the $i_{l}$ th entry as 1 , the $j_{l}$ th entry as -1 , and others as 0 ; otherwise, if $i_{l}=0$, then $\mathbf{e}_{i_{l}, j_{l}}$ is a column vector with the $j_{l}$ th entry as 1 and others as 0 .

Proof. If $i_{l} \neq 0$, the relevant parts of the dynamic equation can be obtained as

$$
\left\{\begin{array}{l}
m_{i_{l}} \ddot{q}_{i_{l}}+\cdots=k_{l s}\left(q_{j_{l}}-q_{i_{l}}\right), \\
m_{j_{l}} \ddot{q}_{j_{l}}+\cdots=-k_{l s}\left(q_{j_{l}}-q_{i_{l}}\right),
\end{array}\right.
$$

where the abridged parts are not affected by the $i_{l}$ th VSS. Therefore, $\quad \mathbf{e}_{i_{1}, j_{l}}=[0,0, \ldots,-1,0, \ldots, 1,0, \ldots, 0]^{T}$, where line $i_{l}$ is -1 and line $j_{l}$ is 1 in this vector.

If $i_{l}=0$, the relevant parts of the dynamic equation can be obtained as

$$
m_{j_{l}} \ddot{q}_{j_{l}}+\cdots=-k_{l s} q_{j_{l}}
$$

where the abridged parts are not affected by the $i_{l}$ th VSS. Therefore, $\mathbf{e}_{i_{l}, j_{l}}=[0,0, \ldots, 1,0, \ldots, 0]^{T}$, where line $j_{l}$ is 1 in this vector.

According to Proposition 1 , if $i_{l} \neq 0, \mathbf{e}_{i_{l}, j_{l}}$ is a column vector with the $i_{l}$ entry as $1, j_{l}$ entry as -1 , and others as 0 ; otherwise, if $i_{l}=0$, then $\mathbf{e}_{i_{l}, j_{l}}$ is a column vector with the $j_{l}$ entry as 1 and others as 0 .

Then, the dynamic equation of the multibody system with $n_{k}$ VSSs can be represented as

$$
\mathbf{M}_{p} \ddot{\mathbf{q}}(t)+\mathbf{C}_{p} \dot{\mathbf{q}}(t)+\mathbf{K}_{p} \mathbf{q}(t)+\sum_{l=1}^{n_{k}} k_{l}(t) \mathbf{e}_{i_{l}, j_{l}} \mathbf{e}_{i_{l}, j_{l}}^{T} \mathbf{q}(t)=0,
$$

where $k_{l}(t)$ is the stiffness of the VSS $l$ and $\mathbf{e}_{i_{l}, j_{l}} \in \mathbf{R}^{N \times 1}$ is the position vector for VSS $l$.

Denote $\mathbf{H}_{k} \in \mathbf{R}^{N \times n_{k}}$ with

$$
H_{k}=\left[e_{i_{1}, j_{1}}, e_{i_{2}, j_{2}}, \ldots, e_{i_{n_{k}}, j_{n_{k}}}\right]
$$

and then, equation (10) can be rewritten as

$$
\mathbf{M}_{p} \ddot{\mathbf{q}}(t)+\mathbf{C}_{p} \dot{\mathbf{q}}(t)+\mathbf{K}_{p} \mathbf{q}(t)+\mathbf{H}_{k} \mathbf{K}_{s}(t) \mathbf{H}_{k}^{T} \mathbf{q}(t)=0,
$$

where $\mathbf{K}_{s}(t)=\operatorname{diag}\left\{k_{1 s}(t), \ldots, k_{n_{k} s}(t)\right\}$.

Therefore,

$$
\ddot{\mathbf{q}}(t)=-\mathbf{M}_{p}^{-1} \mathbf{C}_{p} \dot{\mathbf{q}}(t)-\mathbf{M}_{p}^{-1} \mathbf{K}_{p} \mathbf{q}(t)-\mathbf{M}_{p}^{-1} \mathbf{H}_{k} \mathbf{K}_{s}(t) \mathbf{H}_{k}^{T} \mathbf{q}(t) .
$$

Denoting

$$
\mathbf{x}=\left[\mathbf{q}(t)^{T}, \dot{q}(t)^{T}\right]^{T}
$$


one can obtain the state-space representation of the $N$-DOF system with VSSs as

$$
\left\{\begin{array}{l}
\dot{\mathbf{x}}=\mathbf{A x}+\mathbf{B u}, \\
\mathbf{y}=\mathbf{C x} \\
\mathbf{u}=-\mathbf{\Psi}(t, \mathbf{y})
\end{array}\right.
$$

where

$$
\begin{aligned}
\mathbf{A} & =\left[\begin{array}{cc}
0_{N \times N} & \mathbf{I}_{N \times N} \\
-\mathbf{M}_{p}^{-1} \mathbf{K}_{p} & -\mathbf{M}_{p}^{-1} \mathbf{C}_{p}
\end{array}\right], \\
\mathbf{B} & =\left[\begin{array}{c}
0_{N \times n_{k}} \\
\mathbf{M}_{p}^{-1} \mathbf{H}_{k}
\end{array}\right], \\
\mathbf{C} & =\left[\begin{array}{ll}
\mathbf{H}_{k}^{T} & 0_{n_{k} \times N}
\end{array}\right], \\
\mathbf{\Psi}(t, \mathbf{y}) & =\mathbf{K}_{s}(t) \mathbf{y} .
\end{aligned}
$$

Remark 1. Note that, in [2], the inherently stability of "passive" VSS-based semiactive systems is studied, while in this paper, multibody systems with VSSs are "active." In the following, the passivity of these systems is analyzed via energy consumption.

Note that $\mathbf{H}_{k}$ is a matrix containing pairs (or singleton) of connection points such that the vector of relative displacements across the devices is $\mathbf{q}_{c}=\mathbf{H}_{k}^{T} \mathbf{q}$. Consider a storage function that is equal to the potential energy $E_{p}$ stored in VSSs:

$$
V(t)=\int_{0}^{\mathbf{q}_{c}} \mathbf{q}_{c}^{T} \mathbf{K}_{s}(t) \mathrm{d} \mathbf{q}_{c} .
$$

The derivative of $V(t)$ equals

$$
\begin{aligned}
\dot{V}(t) & =\frac{\partial \int_{0}^{\mathbf{q}_{c}} \mathbf{q}_{c}^{T} \mathbf{K}_{s}(t) \mathrm{d} \mathbf{q}_{c}}{\partial \mathbf{q}_{c}} \cdot \dot{q}_{c}+\frac{\partial \int_{0}^{\mathbf{q}_{c}} \mathbf{q}_{c}^{T} \mathbf{K}_{s}(t) \mathrm{d} \mathbf{q}_{c}}{\partial \mathbf{K}_{s}(t)} \cdot \dot{K}_{s}(t) \\
& =\dot{q}_{c}^{T} \mathbf{K}_{s}(t) \mathbf{q}_{c}+\frac{1}{2} \mathbf{q}_{c}^{T} \dot{K}_{s}(t) \mathbf{q}_{c} .
\end{aligned}
$$

According to Definition 4, for the VSSs-based systems, assume that the output is $\mathbf{y}_{s}=\dot{q}_{c}$, and the external power input $\mathbf{u}_{s}=\mathbf{K}_{s}(t) \mathbf{q}_{c}$. Substituting equation (18) into equation (4), the dissipated power can be solved as follows:

$$
g(t)=-\frac{1}{2} \mathbf{q}_{c}^{T} \dot{K}_{s}(t) \mathbf{q}_{c} .
$$

In [2], to ensure the passivity, the resettable-stiffness spring is proposed. However, the restriction is that the adjustment actions of the spring only take place at the instant that $\mathbf{q}_{c}=0$. In this paper, "active" multibody systems with VSSs are studied. Compared with the restriction to ensure the passivity, it should only satisfy that $\dot{K}_{s}(t)>0$, $\exists t \in[0, T]$, which is common in practice. To the best of our knowledge, this is for the first time in the spring-related research field.
Remark 2. Note that, in Definition 1, the bounds of the sector are better to be constants or diagonal matrices for the absolute stability analysis, and the matrix $\mathbf{K}_{s}(t)$ in equation (15) is always diagonal no matter what the position and number of VSSs are. Therefore, a Lur'e-type form (15) can represent the general multibody systems with VSSs.

The transfer function of equation (15) is

$$
\mathbf{G}(s)=\mathbf{C}(s \mathbf{I}-\mathbf{A})^{-1} \mathbf{B}+\mathbf{D} .
$$

Let

$$
\mathbf{H}(s)=\left[\mathbf{I}+\bar{K}_{s} \mathbf{G}(s)\right]\left[\mathbf{I}+\underline{K}_{s} \mathbf{G}(s)\right]^{-1},
$$

where $\bar{K}_{s}$ and $\underline{K}_{s}$ are the upper and lower bound of the matrix $\mathbf{K}_{s}$.

From Lemma 4 , the transfer function $\mathbf{H}\left(s-\varepsilon_{N}\right)$ is positive real, if there exists a symmetric, positive-definite matrix $\mathbf{X}_{N}$, which satisfies

$$
\left[\begin{array}{cc}
\mathbf{A}_{N}^{T} \mathbf{X}_{N}+\mathbf{X}_{N} \mathbf{A}_{N} & \mathbf{X}_{N} \mathbf{B}_{N}-\mathbf{C}_{N} \\
\mathbf{B}_{N}^{T} \mathbf{X}_{N}-\mathbf{C}_{N}^{T} & -\mathbf{D}_{N}-\mathbf{D}_{N}^{T}
\end{array}\right] \leq 0,
$$

where $\left\{\mathbf{A}_{N}, \mathbf{B}_{N}, \mathbf{C}_{N}, \mathbf{D}_{N}\right\}$ is converted by the transfer function $\mathbf{H}\left(s-\varepsilon_{N}\right)$.

From Lemma 3, the transfer function $\mathbf{H}(s)$ is strictly positive real if there exist some $\varepsilon_{N}$, which satisfies that $\mathbf{H}(s-$ $\left.\varepsilon_{N}\right)$ is positive real.

According to Lemma 1, if the transfer function $\mathbf{H}(s)$ is strictly positive real, the $\mathrm{N}$-DOF multibody system with VSSs is absolutely stable.

\section{Numerical Simulation}

In this section, numerical simulations of the absolute stability of several low-DOF systems are conducted. In Section 4.1 , the absolute stability problem of semiactive control systems with only one VSS is studied. Section 4.2 derives the sufficient conditions for semiactive control systems with several VSSs.

\subsection{Absolute Stability Analysis of the System with One VSS}

Theorem 1. Consider a 1-DOF system with one VSS, seen in Figure 2; the dimensionless dynamic equation is

$$
\ddot{x}_{1}+2 \xi_{1} \omega_{1} \dot{x}_{1}+\left[\omega_{1}^{2}+\omega_{1 s}^{2}(t)\right] x_{1}=0,
$$

where $\quad \xi_{1}=c_{1} /\left(2 \sqrt{k_{1} m_{1}}\right), \quad \omega_{1}=\sqrt{k_{1} / m_{1}}, \quad \omega_{1 s}(t)=$ $\sqrt{k_{1 s}(t) / m_{1}}$, and $0 \leq \underline{\omega}_{1 s} \leq \omega_{1 s}(t) \leq \bar{\omega}_{1 s}\left(m_{1}, c_{1}, k_{1}, k_{1 s}(t)\right.$, and $x_{1}$ represent mass, damping, stiffness, variable stiffness, and displacement of the 1-DOF system, respectively). If it satisfies equation (24), the 1-DOF system with one VSS is absolutely stable.

$$
a>0 \text {, or }\left\{\begin{array}{l}
a \leq 0, \\
4\left(\omega_{1}^{2}+\underline{\omega}_{1 s}^{2}\right)\left(\omega_{1}^{2}+\bar{\omega}_{1 s}^{2}\right)-a^{2}>0,
\end{array}\right.
$$

where $a=\left(4 \xi_{1}^{2}-2\right) \omega_{1}^{2}-\left(\underline{\omega}_{1 s}^{2}+\bar{\omega}_{1 s}^{2}\right)$. 


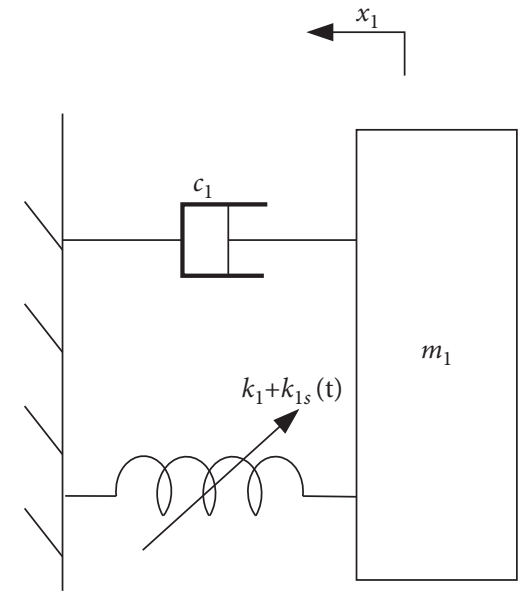

FIgURE 2: A 1-DOF model with a VSS.

Proof. Denote $\mathbf{x}_{1}=\left[x_{1}, \dot{x}_{1}\right]^{T}$; equation (23) can be converted to

$$
\left\{\begin{array}{l}
\dot{\mathbf{x}}_{1}=\mathbf{A}_{1} \mathbf{x}_{1}+\mathbf{B}_{1} u_{1} \\
\mathbf{y}_{1}=\mathbf{C}_{1} \mathbf{x}_{1} \\
\mathbf{u}_{1}=-\Psi_{1}\left(t, y_{1}\right)
\end{array}\right.
$$

where

$$
\begin{aligned}
\mathbf{A}_{1} & =\left[\begin{array}{cc}
0 & 1 \\
-\omega_{1}^{2} & -2 \xi_{1} \omega_{1}
\end{array}\right], \\
\mathbf{B}_{1} & =\left[\begin{array}{l}
0 \\
1
\end{array}\right], \\
\mathbf{C}_{1} & =\left[\begin{array}{ll}
1 & 0
\end{array}\right], \\
\Psi_{1}\left(t, y_{1}\right) & =\omega_{1 s}^{2} y_{1} .
\end{aligned}
$$

The transfer function of equation (25) is

$$
G_{1}(s)=\mathbf{C}_{1}\left(s \mathbf{I}-\mathbf{A}_{1}\right)^{-1} \mathbf{B}_{1}=\frac{1}{s^{2}+2 \xi_{1} \omega_{1} s+\omega_{1}^{2}} .
$$

Let

$$
\begin{aligned}
H_{1}(s) & =\left[1+\bar{\omega}_{1 s}^{2} G_{1}(s)\right]\left[1+\bar{\omega}_{1 s}^{2} G_{1}(s)\right]^{-1} \\
& =1+\frac{\bar{\omega}_{1 s}^{2}-\underline{\omega}_{1 s}^{2}}{s^{2}+2 \xi_{1} \omega_{1} s+\omega_{1}^{2}+\underline{\omega}_{1 s}^{2}} .
\end{aligned}
$$

The transfer function $H_{1}(s)$ can be converted to statespace realization $\left(\mathbf{A}_{\alpha}, \mathbf{B}_{\alpha}, \mathbf{C}_{\alpha}, \mathbf{D}_{\alpha}\right)$, where

$$
\begin{aligned}
& \mathbf{A}_{\alpha}=\left(\begin{array}{cc}
0 & 1 \\
-\omega_{1}^{2}-\underline{\omega}_{1 s}^{2} & -2 \xi_{1} \omega_{1}
\end{array}\right), \\
& \mathbf{B}_{\alpha}=\left(\begin{array}{c}
0^{2} \\
\bar{\omega}_{1 s}-\underline{\omega}_{1 s}^{2}
\end{array}\right), \\
& \mathbf{C}_{\alpha}=\left(\begin{array}{ll}
1 & 0
\end{array}\right), \\
& \mathbf{D}_{\alpha}=1 .
\end{aligned}
$$

It is clear that all the eigenvalues of $\mathbf{A}_{\alpha}$ have negative real part. Then, according to Lemma 6, all elements of $H_{1}(s)$ are analytic in $\operatorname{Re}(s) \geq 0$, which satisfies list 1 of Lemma 5 .

According to list 1 of Lemma 5, it is obvious that all elements of $H_{1}(s)$ are analytic in $\operatorname{Re}(s) \geq 0$. According to list 2 of Lemma 5 ,

$$
H_{1}(j \omega)+H_{1}^{T}(-j \omega)=1+\frac{\left(\bar{\omega}_{1 s}^{2}-\underline{\omega}_{1 s}^{2}\right)\left(-\omega^{2}+\omega_{1}^{2}+\underline{\omega}_{1 s}^{2}\right)}{\left(-\omega^{2}+\omega_{1}^{2}+\underline{\omega}_{1 s}^{2}\right)^{2}+\left(2 \xi_{1} \omega_{1} \omega\right)^{2}} .
$$

In order to satisfy that $H_{1}(j \omega)+H_{1}^{T}(-j \omega)>0, \forall \omega \in R$, it can be obtained that

$$
f(\omega)>0, \quad \forall \omega \in R,
$$

where $\quad f(\omega)=\omega^{4}+\left[\left(2 \xi_{1} \omega_{1}\right)^{2}-2\left(\omega_{1}^{2} \quad+\underline{\omega}_{1 s}^{2}\right)-\left(\bar{\omega}_{1 s}^{2}-\right.\right.$ $\left.\left.\underline{\omega}_{1 s}^{2}\right)\right] \omega^{2}+\left(\omega_{1}^{2}+\underline{\omega}_{1 s}^{2}\right)\left(\omega_{1}^{2}+\bar{\omega}_{1 s}^{2}\right)$.

According to the position of the symmetry axis, the following inequality can be obtained:

$$
\left\{\begin{array}{l}
\left(2 \xi_{1} \omega_{1}\right)^{2}-2\left(\omega_{1}^{2}+\underline{\omega}_{1 s}^{2}\right)-\left(\bar{\omega}_{1 s}^{2}-\underline{\omega}_{1 s}^{2}\right) \geq 0, \\
f(0)>0, \\
\underline{\omega}_{1 s}<\bar{\omega}_{1 s},
\end{array}\right.
$$

or

$$
\left\{\begin{array}{l}
\left(2 \xi_{1} \omega_{1}\right)^{2}-2\left(\omega_{1}^{2}+\underline{\omega}_{1 s}^{2}\right)-\left(\bar{\omega}_{1 s}^{2}-\underline{\omega}_{1 s}^{2}\right)<0, \\
f_{\min }(\omega)>0 \\
\underline{\omega}_{1 s}<\bar{\omega}_{1 s} .
\end{array}\right.
$$

By simplifying the inequalities equations (32) and (33), the following sufficient condition can be obtained:

$$
\begin{aligned}
a>0, & \text { or }\left\{\begin{array}{l}
a \leq 0, \\
4\left(\omega_{1}^{2}+\underline{\omega}_{1 s}^{2}\right)\left(\omega_{1}^{2}+\bar{\omega}_{1 s}^{2}\right)-a^{2}>0,
\end{array}\right.
\end{aligned}
$$

where $a=4\left(\omega_{1}^{2}+\underline{\omega}_{1 s}^{2}\right)\left(\omega_{1}^{2}+\bar{\omega}_{1 s}^{2}\right)$.

Therefore, in order to satisfy that $H_{1}(j \omega)+H_{1}^{T}(-j \omega)$ $>0, \forall \omega \in R$, equation (34) should be satisfied.

According to list 3 of Lemma 5, note that $D=1$ in $H_{1}(s)$, and then

$$
\lim _{\omega \longrightarrow \infty}\left[H_{1}(j \omega)+H_{1}^{T}(-j \omega)\right]=2>0 .
$$

Therefore, list 3 of Lemma 5 is obviously satisfied in all conditions.

In summary, if equation (34) is satisfied, $H_{1}(s)$ is a strictly positive real matrix.

According to Lemma 1, note that $\Psi \in\left[\underline{\omega}_{1 s}^{2}, \bar{\omega}_{1 s}^{2}\right]$, and

$$
H_{1}(s)=\left[1+\bar{\omega}_{1 s}^{2} G_{1}(s)\right]\left[1+\underline{\omega}_{1 s}^{2} G_{1}(s)\right]^{-1},
$$

is strictly positive real. Therefore, the 1-DOF system with one VSS is absolutely stable. 
Example 1. Consider a 1-DOF system, seen in Figure 2, and choose $\omega_{1}=1, \xi_{1}=2, \underline{\omega}_{1 s}=1$. In the following, the range of $\bar{\omega}_{1 s}$ is calculated by equation (32) or (33). From equation (32), the range of $\bar{\omega}_{1 s}$ is calculated as $(1, \sqrt{13}]$, and from equation (33), the range is $(\sqrt{13}, 5.321]$. Therefore, $\bar{\omega}_{1 s}$ can be set from 1 to 5.321 to ensure the absolute stability of the 1DOF system. Figure 3 shows the Nyquist plot and disk plot of the 1-DOF system; the system is absolutely stable, according to Lemma 2.

Apart from verification in the frequency domain, timedomain simulation is also considered as follows. Two kinds of nonlinearities are considered. For the first one, the selected $\omega_{1 s}$ is given in equation (37), which is in the range $\omega_{1 s} \in(1,5.321]$. The other selected $\bar{\omega}_{1 s}$ is given in equation (38), which is out of the range $\omega_{1 s} \in(1,5.321]$.

$$
\begin{aligned}
& \omega_{1 s}=\sin (t)+2, \\
& \omega_{1 s}= \begin{cases}7, & x_{1}(t) \dot{x}_{1}(t)>0, \\
14, & x_{1}(t) \dot{x}_{1}(t) \leq 0 .\end{cases}
\end{aligned}
$$

Time-domain simulation is shown in Figure 4 . Figure 4(a) shows the phase trajectory of state $x_{1}$ when the selected $\bar{\omega}_{1 s}$ is in the stable range. The phase trajectory from a specific initial condition $\left(x_{1}(0)=1, \dot{x}_{1}(0)=0\right)$, which converge to the origin, indicates the stability of the system. Figure 4(b) shows the phase trajectory of state $x_{1}$ when the selected $\bar{\omega}_{1 s}$ is out of the stable range. The phase trajectory from a specific initial condition $\left(x_{1}(0)=1, \dot{x}_{1}(0)=0\right)$, which is divergent, indicates the instability of the system.

Remark 3. Note that Lemmas 3 and 4 can also verify whether $H_{1}(s)$ is strictly positive real, apart from Lemma 5 . If there exist positive $\varepsilon_{1}$ and symmetric, positive-definite matrix $\mathbf{X}_{1}$ satisfying equation (39), the 1-DOF system with one VSS is absolutely stable.

$$
\left[\begin{array}{cc}
\mathbf{A}_{11}^{T} \mathbf{X}_{1}+\mathbf{X}_{1} \mathbf{A}_{11} & \mathbf{X}_{1} \mathbf{B}_{11}-\mathbf{C}_{11} \\
\mathbf{B}_{11}^{T} \mathbf{X}_{1}-\mathbf{C}_{11}^{T} & -\mathbf{D}_{11}-\mathbf{D}_{11}^{T}
\end{array}\right] \leq 0,
$$

where $\left(\mathbf{A}_{11}, \mathbf{B}_{11}, \mathbf{C}_{11}, \mathbf{D}_{11}\right)$ is converted by $H_{1}\left(s-\varepsilon_{1}\right)$.

In the following, numerical simulation is given to compare the conservatism. The values of $\underline{\omega}_{1 s}, \omega_{1}$, and $\xi_{1}$ are the same as Example 1. Equation (33) is feasible when $\bar{\omega}_{1 s}$ varies from 1 to 5.3. Figure 5 shows the Nyquist plot and disk plot of the 1-DOF system; the system is absolutely stable, according to Lemma 2.

Note that the range of $\omega_{1 s}$ is smaller than that in Example 1; the LMI condition in this remark is more conservative than that in Theorem 1 . Therefore, it is better to analyze the absolute stability of a 1-DOF system with variable stiffness spring by equation (24) in Theorem 1 .

Example 2. A comparison between the method proposed in this paper and the traditional stability analysis method with a quadratic Lyapunov function for the 1-DOF system shown in Figure 2 is conducted. The values of $\omega_{1}$ and $\xi_{1}$ are the same as those in Example 1, and a VSS with a periodic stiffness, i.e.,

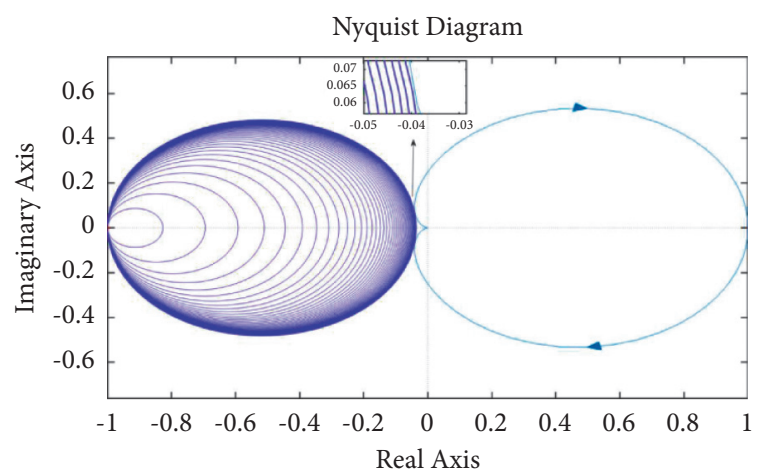

Figure 3: The Nyquist diagram with $\bar{\omega}_{1 s} \in(1,5.321]$.

$$
\omega_{1 s}^{2}=k_{\alpha}+k_{\beta} \sin (t),
$$

which can be found in robot manipulators such as in [25], is adopted.

Let $k_{\beta}=0.5$ and $k_{\alpha} \geq 0.5$. For the traditional stability analysis method with a quadratic Lyapunov function, one can select a quadratic Lyapunov function as

$$
V(t)=\frac{1}{2} \mathbf{x}_{1}^{T} \mathbf{Q} \mathbf{x}_{1},
$$

where $\mathbf{Q}=\left[\begin{array}{cc}1 & 1 / 2 \\ 1 / 2 & 1\end{array}\right]$. It can be checked that if $1 / 2 \leq k_{\alpha} \leq(2+\sqrt{21}) / 2$, then $V>0$ and $\dot{V} \leq 0$, indicating that the 1 -DOF system is stable.

By using the method proposed in this paper, from equation (24), one obtains that the 1-DOF system with a VSS is always stable whenever $\omega_{1 s}$ varies in the interval $[0,2 \sqrt{6}]$. This means that the stability condition obtained by the traditional quadratic Lyapunov function method only holds for specific cases such as the periodic variable stiffness in equation (40), while the condition obtained by the method proposed in this paper is applicable for any type of VSS's variable stiffness within a sector. To further show this fact, the following variable stiffnesses are considered:

$$
\begin{aligned}
& \omega_{1 s}^{2}=3+0.5 \sin (t), \\
& \omega_{1 s}^{2}=10+0.5 \sin (t) .
\end{aligned}
$$

Figure 6(a) shows the sector, seen as equation (44), induced by a VSS. The red solid lines represent the bound of the sector $[0,24]$. The black dashed line and the blue dotted line represent the periodic variable stiffness, (42) and (43), respectively.

$$
\Psi_{1}\left(t, y_{1}\right)=\omega_{1 s}^{2} y_{1}, \quad y_{1} \in[-1,1] .
$$

It is clear that the case with equation (42) is stable by using the quadratic Lyapunov function in equation (41). However, for the case with equation (43), the stability cannot be verified by using the quadratic Lyapunov function in equation (41), while since it is within the sector as shown in Figure 6(a), the stability of the case with equation (43) can be checked by using the method proposed in this paper, where 


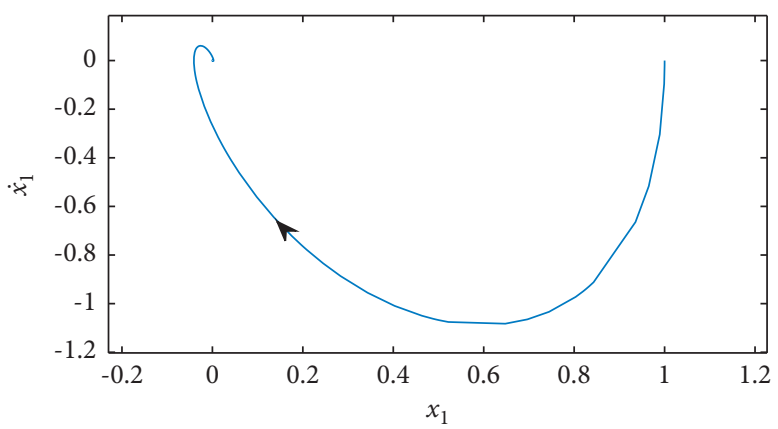

(a)

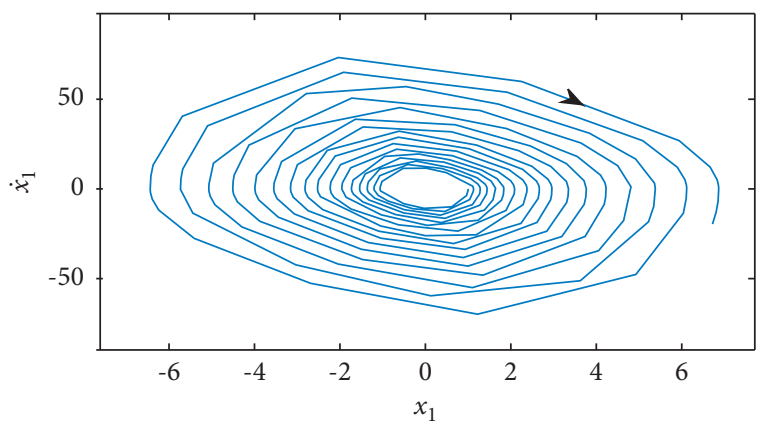

(b)

FIGURE 4: The phase portrait of a 1-DOF systems with different nonlinearities in the feedback path. (a) The phase trajectory of state $x_{1}$ with the selected $\bar{\omega}_{1 s}$ in the stable range. (b) The phase trajectory of state $x_{1}$ with the selected $\bar{\omega}_{1 s}$ out of the stable range.

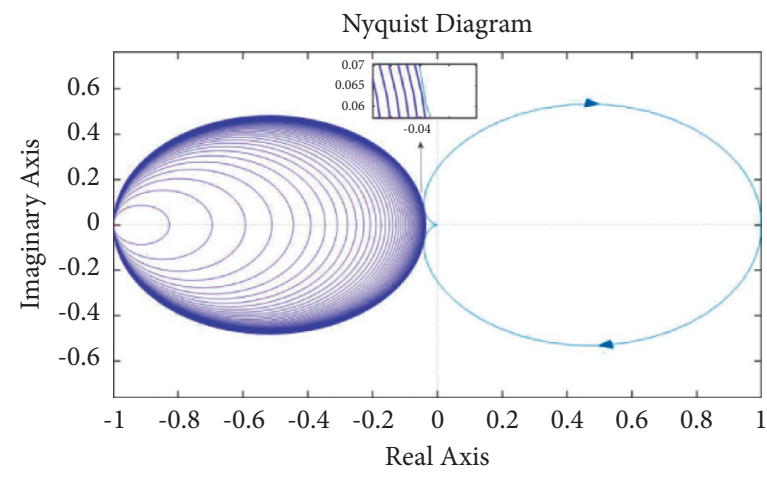

FIgURE 5: The Nyquist diagram with $\bar{\omega}_{1 s} \in(1,5.3]$.

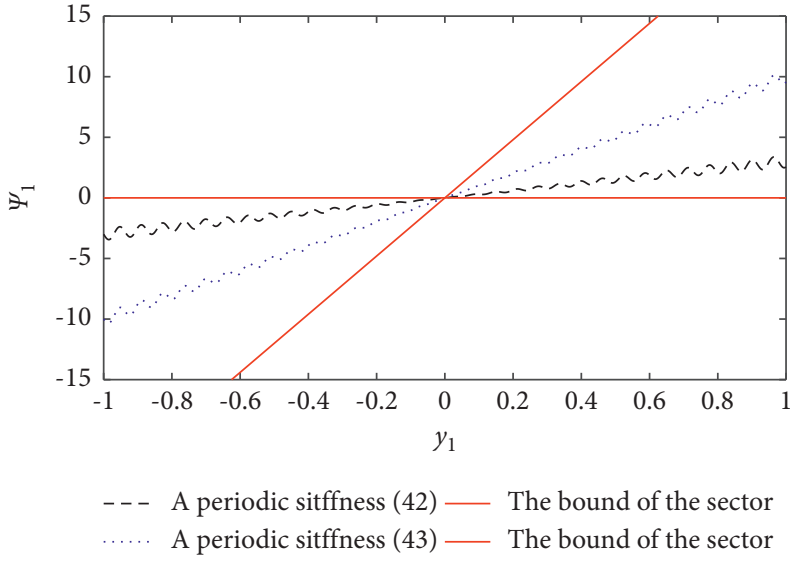

(a)

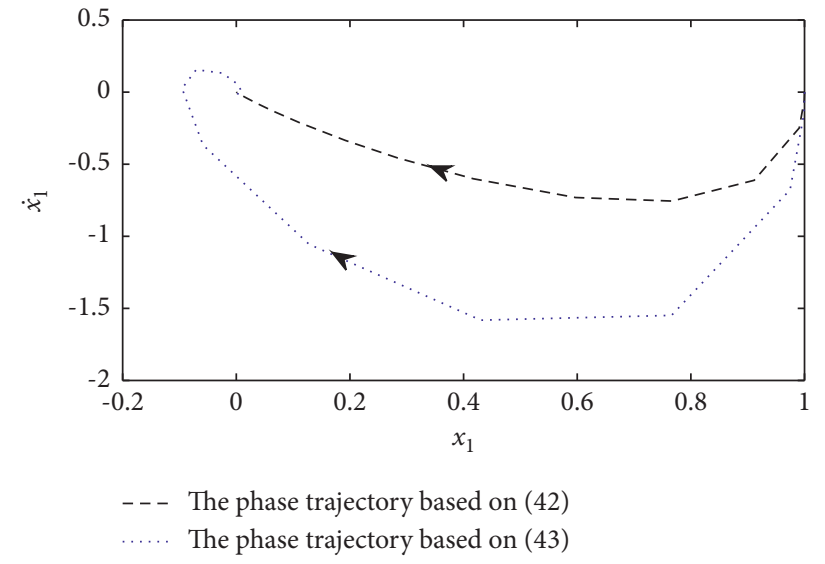

(b)

Figure 6: A comparison between the method proposed in this paper and the traditional quadratic Lyapunov function method. (a) The sector region induced by a VSS. (b) The phase portrait of a 1-DOF system with a VSS.

Figure 6(b) shows that both cases are stable. This example demonstrates that the method proposed in this paper is applicable for a group of nonlinearities within a certain sector, instead of a specific nonlinearity.

\subsection{Absolute Stability Analysis of the Systems with VSSs}

Theorem 2. Consider a 2-DOF system with VSSs, seen in Figure 7; the dimensionless dynamic equation is 


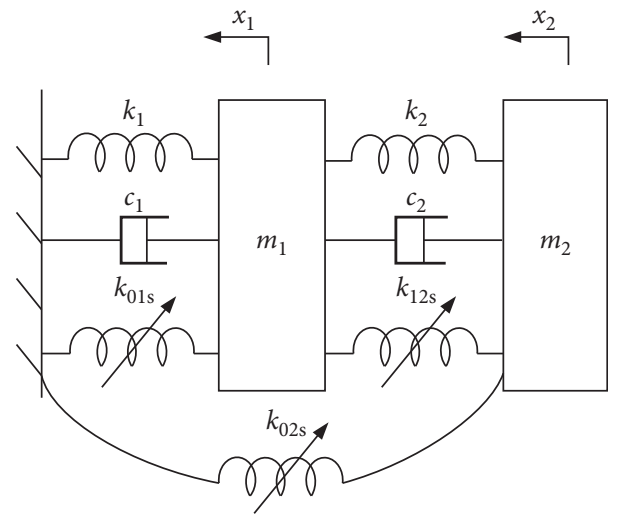

Figure 7: A 2-DOF model with VSSs.

$$
\left\{\begin{array}{l}
\ddot{x}_{1}+\left(2 \xi_{1} \omega_{1}+2 \xi_{2} \omega_{2} \alpha_{1}\right) \dot{x}_{1}+\left(\omega_{1}^{2}+\omega_{2}^{2} \alpha_{1}\right) x_{1}+\left[\omega_{01 s}^{2}(t)+\omega_{12 s}^{2}(t)\right] x_{1} \\
-2 \xi_{2} \omega_{2} \alpha_{1} \dot{x}_{2}-\omega_{2}^{2} \alpha_{1} x_{2}-\omega_{12 s}^{2}(t) x_{2}=0 \\
\alpha_{1} \ddot{x}_{2}+2 \xi_{2} \omega_{2} \alpha_{1} \dot{x}_{2}+\omega_{2}^{2} \alpha_{1} x_{2}+\left[\omega_{12 s}^{2}(t)+\omega_{02 s}^{2}(t)\right] x_{2} \\
-2 \xi_{2} \omega_{2} \alpha_{1} \dot{x}_{1}-\omega_{2}^{2} \alpha_{1} x_{1}-\omega_{12 s}^{2}(t) x_{1}=0
\end{array}\right.
$$

where $\xi_{1}=c_{1} /\left(2 \sqrt{k_{1} m_{1}}\right), \omega_{1}=\sqrt{k_{1} / m_{1}}, \xi_{2}=c_{2} /\left(2 \sqrt{k_{2} m_{2}}\right)$, $\omega_{2}=\sqrt{k_{2} / m_{2}}, \quad \alpha_{1}=m_{2} / m_{1}, \quad \omega_{01 s}(t)=\sqrt{k_{01 s}(t) / m_{1}}, \quad \omega_{12 s}$ $(t)=\sqrt{k_{12 s}(t) / m_{1}}, \quad \omega_{02 s}(t)=\sqrt{k_{02 s}(t) / m_{1}}$, and $\omega_{01 s}\left(\omega_{12 s}\right.$, or $\left.\omega_{02 s}\right)$ varies from $\underline{\omega}_{01 s}\left(\underline{\omega}_{12 s}\right.$, or $\left.\underline{\omega}_{02 s}\right)$ to $\bar{\omega}_{01 s}\left(\bar{\omega}_{12 s}\right.$, or $\left.\bar{\omega}_{02 s}\right)$. $m_{1}\left(m_{2}\right), c_{1}\left(c_{2}\right), k_{1}\left(k_{2}\right), k_{01 s}(t)\left(k_{12 s}(t), k_{02 s}(t)\right)$, and $x_{1}\left(x_{2}\right)$ represent mass, damping, stiffness, variable stiffness, and displacement of the 2-DOF system, respectively. If there exist positive $\varepsilon_{2}$ and symmetric, positive-definite matrix $\mathbf{X}_{2}$ satisfying equation (46), the 2-DOF system with VSSs is absolutely stable.

$$
\left[\begin{array}{cc}
\mathbf{A}_{21}^{T} \mathbf{X}_{2}+\mathbf{X}_{2} \mathbf{A}_{21} & \mathbf{X}_{2} \mathbf{B}_{21}-\mathbf{C}_{21} \\
\mathbf{B}_{21}^{T} \mathbf{X}_{2}-\mathbf{C}_{21}^{T} & -\mathbf{D}_{21}-\mathbf{D}_{21}^{T}
\end{array}\right] \leq 0,
$$

where $\left\{\mathbf{A}_{21}, \mathbf{B}_{21}, \mathbf{C}_{21}, \mathbf{D}_{21}\right\}$ is converted by $\mathbf{H}_{2}\left(s-\varepsilon_{2}\right)$.

Proof. Denote $\mathbf{x}_{1}=\left[x_{1}, x_{2}, \dot{x}_{1}, \dot{x}_{2}\right]^{T}$; equation (45) can be converted to

$$
\left\{\begin{array}{l}
\dot{\mathbf{x}}_{2}=\mathbf{A}_{2} \mathbf{x}_{2}+\mathbf{B}_{2} \mathbf{u}_{2}, \\
\mathbf{y}_{2}=\mathbf{C}_{2} \mathbf{x}_{2}, \\
\mathbf{u}_{2}=-\Psi_{2}\left(t, \mathbf{y}_{2}\right),
\end{array}\right.
$$

where

$$
\begin{aligned}
& \mathbf{A}_{2}=\left[\begin{array}{cccc}
0 & 0 & 1 & 0 \\
0 & 0 & 0 & 1 \\
-\omega_{2}^{2} \alpha_{1}-\omega_{1}^{2} & \omega_{2}^{2} \alpha_{1} & -2 \xi_{2} \omega_{2} \alpha_{1}-2 \xi_{1} \omega_{1} & 2 \xi_{2} \omega_{2} \alpha_{1} \\
\omega_{2}^{2} & -\omega_{2}^{2} & 2 \xi_{2} \omega_{2} & -2 \xi_{2} \omega_{2}
\end{array}\right], \\
& \mathbf{B}_{2}=\left[\begin{array}{ccc}
0 & 0 & 0 \\
0 & 0 & 0 \\
-1 & 1 & 0 \\
0 & -1 / \alpha_{1} & -1 / \alpha_{1}
\end{array}\right], \\
& \mathbf{C}_{2}=\left[\begin{array}{cccc}
1 & 0 & 0 & 0 \\
-1 & 1 & 0 & 0 \\
0 & 1 & 0 & 0
\end{array}\right],
\end{aligned}
$$$$
\Psi_{2}\left(t, \mathbf{y}_{2}\right)=\operatorname{diag}\left\{\omega_{01 s}^{2}, \omega_{12 s}^{2}, \omega_{02 s}^{2}\right\} \mathbf{y}_{2}
$$

The transfer function of equation (47) is 


$$
\begin{aligned}
\mathbf{G}_{2}(s) & =\mathbf{C}_{1}\left(s \mathbf{I}-\mathbf{A}_{2}\right)^{-1} \mathbf{B}_{2} \\
& =\left[\begin{array}{ccc}
-\frac{s^{2}+2 \omega_{2} \xi_{2} s+\omega_{2}^{2}}{\operatorname{den}} & \frac{s^{2}}{\operatorname{den}} & -\frac{2 \omega_{2} \xi_{2} s+\omega_{2}^{2}}{\operatorname{den}} \\
* & -\frac{\left(1+\alpha_{1}\right) s^{2}+2 \omega_{1} \xi_{1} s+\omega_{1}^{2}}{\alpha_{1} \cdot \operatorname{den}} & -\frac{s^{2}+2 \omega_{1} \xi_{1} s+\omega_{1}^{2}}{\alpha_{1} \cdot \operatorname{den}} \\
* & * & -\frac{s^{2}+\left(2 \omega_{1} \xi_{1}+2 \alpha_{1} \omega_{2} \xi_{2}\right) s+\omega_{1}^{2}+\alpha_{1} \omega_{2}^{2}}{\alpha_{1} \cdot \operatorname{den}}
\end{array}\right],
\end{aligned}
$$

where * denotes the symmetrical element, and

$$
\operatorname{den}=s^{4}+\left(2 \omega_{1} \xi_{1}+2 \omega_{2} \xi_{2}+2 \omega_{2} \xi_{2} \alpha_{1}\right) s^{3}+\left(\omega_{1}^{2}+\omega_{2}^{2}+\omega_{2}^{2} \alpha_{1}+4 \omega_{1} \omega_{2} \xi_{1} \xi_{2}\right) s^{2}+\left(2 \omega_{1}^{2} \omega_{2} \xi_{2}+2 \omega_{1} \omega_{2}^{2} \xi_{1}\right) s+\omega_{1}^{2} \omega_{2}^{2}
$$

Then

$$
\mathbf{H}_{2}(s)=\left[\mathbf{I}+\mathbf{K}_{22} \mathbf{G}_{2}(s)\right]\left[\mathbf{I}+\mathbf{K}_{21} \mathbf{G}_{2}(s)\right]^{-1},
$$

where $\quad \mathbf{K}_{21}=\operatorname{diag}\left\{\underline{\omega}_{01 s}^{2}, \underline{\omega}_{12 s}^{2}, \underline{\omega}_{02 s}^{2}\right\} \quad$ and $\quad \mathbf{K}_{22}=\operatorname{diag}$ $\left\{\bar{\omega}_{01 s}^{2}, \bar{\omega}_{12 s}^{2}, \bar{\omega}_{02 s}^{2}\right\}$. Equation (51) can be converted to the state space $\left(\mathbf{A}_{21}, \mathbf{B}_{21}, \mathbf{C}_{21}, \mathbf{D}_{21}\right)$.

From Lemma 3, the transfer function $\mathbf{H}_{2}(s)$ is strictly positive real if there exist some $\varepsilon_{2}$, which satisfies that $\mathbf{H}_{2}(s-$ $\left.\varepsilon_{2}\right)$ is positive real.

From Lemma 4, the transfer function $\mathbf{H}_{2}\left(s-\varepsilon_{2}\right)$ is positive real if there exists a symmetric, positive-definite matrix $\mathbf{X}_{2}$, which satisfies

$$
\left[\begin{array}{cc}
\mathbf{A}_{21}^{T} \mathbf{X}_{2}+\mathbf{X}_{2} \mathbf{A}_{21} & \mathbf{X}_{2} \mathbf{B}_{21}-\mathbf{C}_{21} \\
\mathbf{B}_{21}^{T} \mathbf{X}_{2}-\mathbf{C}_{21}^{T} & -\mathbf{D}_{21}-\mathbf{D}_{21}^{T}
\end{array}\right] \leq 0 .
$$

Example 3. Choose $\omega_{1}=2, \xi_{1}=6, \omega_{2}=3, \xi_{2}=7, \alpha_{1}=3$, $\underline{\omega}_{01 s}=0, \bar{\omega}_{01 s}=3, \underline{\omega}_{12 s}=0, \bar{\omega}_{12 s}=2, \underline{\omega}_{02 s}=0, \bar{\omega}_{02 s}=1$, and $\varepsilon_{2}=0.1$; the symmetric and positive-definite matrix $\mathbf{X}_{2}$ is calculated by the standard numerical simulation toolbox.

$$
\mathbf{X}_{2}=\left[\begin{array}{cccc}
14.9948 & -9.8401 & 1.1148 & 0.4961 \\
* & 93.1342 & -25.2808 & -8.2322 \\
* & * & 34.7102 & 10.1715 \\
* & * & * & 3.1878 \\
* & * & * & * \\
* & * & * & * \\
* & * & * & * \\
* & * & * & *
\end{array}\right.
$$

$\left.\begin{array}{cccc}-121.9984 & 22.6820 & -0.4832 & -1.3486 \\ -43.7687 & -14.3113 & -4.82 & -7.2607 \\ 22.116 & 1.4992 & 4.415 & 1.9909 \\ 6.141 & 0.7426 & 0.9826 & -0.142 \\ 1176.4 & -186.7525 & 10.925 & 22.7945 \\ * & 38.3025 & -11.2653 & -6.8299 \\ * & * & 106.2525 & 46.7287 \\ * & * & * & 24.697\end{array}\right]$

According to Theorem 2, if there exist some $\varepsilon_{2}>0$ and a symmetric and positive-definite matrix $\mathbf{X}_{2}$, the 2-DOF system with VSSs is absolutely stable.

Apart from verification in the frequency domain, timedomain simulation is also considered as follows. Two kinds of nonlinearities are considered. For the first one, the selected $\omega_{01 s}, \omega_{12 s}$, and $\omega_{02 s}$ are given in equation (54), which are in the ranges $\omega_{01 s} \in[0,3], \omega_{12 s} \in[0,2]$, and $\omega_{02 s} \in[0,1]$. The other selected $\omega_{01 s}, \omega_{12 s}$, and $\omega_{02 s}$ are given in equation (55), which are out of the ranges $\omega_{01 s} \in[0,3], \omega_{12 s} \in[0,2]$, and $\omega_{02 s} \in[0,1]$.

In the following, a time-domain simulation is conducted, similar to that in Example 1. The values of $\omega_{01 s}, \omega_{12 s}$, and $\omega_{02 s}$ are, respectively, selected as equations (54) and (55): 


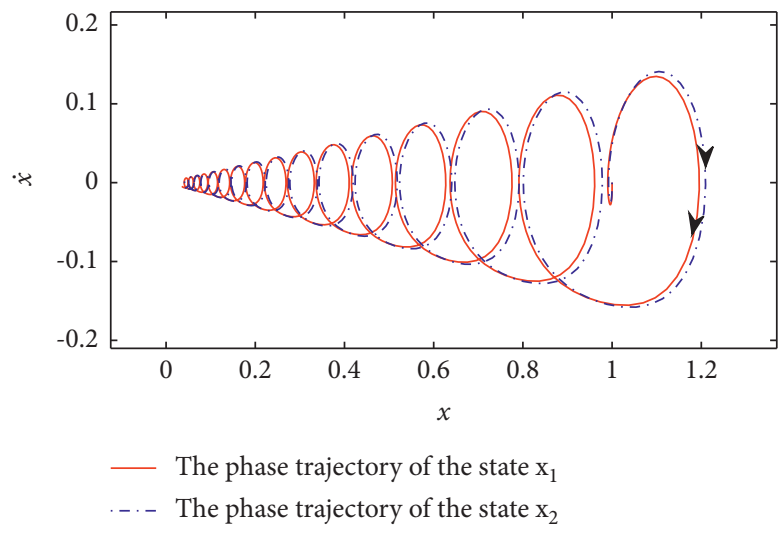

(a)

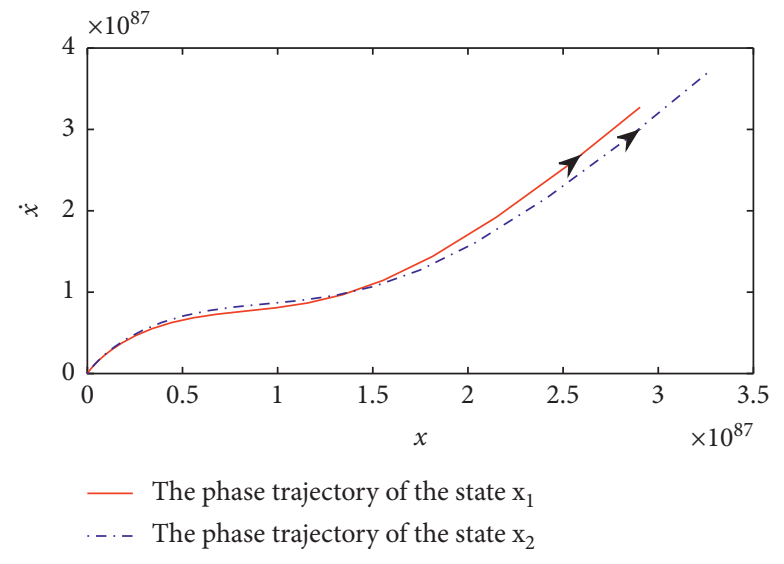

(b)

FIgURE 8: The phase portrait of a 2-DOF system with different nonlinearities in the feedback path. (a) The phase trajectory with the selected values in the stable ranges. (b) The phase trajectory with the selected values out of the stable ranges.

$$
\begin{aligned}
& \left\{\begin{array}{l}
\omega_{01 s}=\sin (t)+1.5 \\
\omega_{12 s}=0.5 \sin (t)+0.6 \\
\omega_{02 s}=0.1 \sin (t)+1.2
\end{array}\right. \\
& \left\{\begin{array}{l}
\omega_{01 s}=\sin (t)+4 \\
\omega_{12 s}=\sin (t)+4 \\
\omega_{02 s}=\sin (t)+2
\end{array}\right.
\end{aligned}
$$

Numerical simulation is shown in Figure 8, where similar results as Example 1 can be found. The system can be stable when the selected values are in the stable ranges, as shown in Figure 8(a). However, when the selected values are out of the stable ranges, the system will be unstable, as shown in Figure 8(b).

\section{Conclusions}

In this paper, the inherent stability problem for multibody systems with VSSs was investigated. Based on the feature that the inherent stability implied the stability for a group of control laws within certain sectors, absolute stability theory was adopted. The general $N$-DOF multibody systems with VSSs were formulated in a Lur'e form, and the circle criterion method was employed to obtain sufficient conditions for the inherent stability of the systems. $\mathrm{Nu}$ merical examples based typical low-DOF systems were conducted to show the effectiveness of the proposed conditions in this paper. Furthermore, the results of this paper can contribute to adjusting the bounds of the stiffness of VSSs to ensure the inherent stability and can also be regarded as a guidance for designing VSSs-based mechanical control systems.

\section{Data Availability}

The data used to support the findings of this study are included within the article.

\section{Conflicts of Interest}

The authors declare that they have no conflicts of interest.

\section{Acknowledgments}

This research was partially supported by the National Natural Science Foundation of China under Grant 62173125, the National Natural Science Foundation of China-State Grid Joint Fund for Smart Grid under Grant U1966202, the Fundamental Research Funds for the Central Universities of China under Grant B210202058, and Postgraduate Research \& Practice Innovation Program of Jiangsu Province under Grant KYCX21_0481.

\section{References}

[1] M. D. Symans and M. C. Constantinou, "Semi-active control systems for seismic protection of structures: a state-of-the-art review," Engineering Structures, vol. 21, no. 6, pp. 469-487, 1999.

[2] H. Garrido, O. Curadelli, and D. Ambrosini, "On the assumed inherent stability of semi-active control systems," Engineering Structures, vol. 159, pp. 286-298, 2018.

[3] T. W. Secord and H. H. Asada, "A variable stiffness PZT actuator having tunable resonant frequencies," IEEE Transactions on Robotics, vol. 26, no. 6, pp. 993-1005, 2010.

[4] T. Kobori, M. Takahashi, T. Nasu, N. Niwa, and K. Ogasawara, "Seismic response controlled structure with Active Variable Stiffness system," Earthquake Engineering \& Structural Dynamics, vol. 22, no. 11, pp. 925-941, 1993.

[5] G.-L. Lin, C.-C. Lin, B.-C. Chen, and T.-T. Soong, "Vibration control performance of tuned mass dampers with resettable variable stiffness," Engineering Structures, vol. 83, pp. 187-197, 2015.

[6] S. Sun, J. Yang, W. Li, H. Deng, H. Du, and G. Alici, "Development of a novel variable stiffness and damping magnetorheological fluid damper," Smart Materials and Structures, vol. 24, no. 8, Article ID 085021, 2015.

[7] D. Ning, H. Du, S. Sun, W. Li, N. Zhang, and M. Dong, "A novel electrical variable stiffness device for vehicle seat 
suspension control with mismatched disturbance compensation," IEEE/ASME Transactions on Mechatronics, vol. 24, no. 5, pp. 2019-2030, 2019.

[8] Z. Guo, Y. Pan, T. Sun, Y. Zhang, and X. Xiao, "Adaptive neural network control of serial variable stiffness actuators," Complexity, vol. 2017, Article ID 5361246, 9 pages, 2017.

[9] C. Ji, M. Kong, and R. Li, "Weight lifting trajectory optimization for variable stiffness actuated robot," Complexity, vol. 2019, Article ID 4872189, 12 pages, 2019.

[10] B. F. Spencer and S. Nagarajaiah, "State of the art of structural control," Journal of Structural Engineering, vol. 129, no. 7, pp. 845-856, 2003.

[11] Y. Liu, H. Matsuhisa, and H. Utsuno, "Semi-active vibration isolation system with variable stiffness and damping control," Journal of Sound and Vibration, vol. 313, no. 1-2, pp. 16-28, 2008.

[12] O. M. Anubi and C. Crane, "A new semiactive variable stiffness suspension system using combined skyhook and nonlinear energy sink-based controllers," IEEE Transactions on Control Systems Technology, vol. 23, no. 3, pp. 937-947, 2014.

[13] B. D. O. Anderson, "The small-gain theorem, the passivity theorem and their equivalence," Journal of the Franklin Institute, vol. 293, no. 2, pp. 105-115, 1972.

[14] M. Corless and G. Leitmann, "Destabilization via active stiffness," Dynamics and Control, vol. 7, no. 3, pp. 263-268, 1997.

[15] W. Kim, C. M. Kang, Y. S. Son, and C. C. Chung, "Nonlinear backstepping control design for coupled nonlinear systems under external disturbances," Complexity, vol. 2019, Article ID 7941302, 13 pages, 2019.

[16] M. Cai, Z. Xiang, and J. Guo, “Adaptive finite-time faulttolerant consensus protocols for multiple mechanical systems," Journal of the Franklin Institute, vol. 353, no. 6, pp. 1386-1408, 2016.

[17] M. Cai, Z. Xiang, and J. Guo, "Adaptive finite-time control for uncertain nonlinear systems with application to mechanical systems," Nonlinear Dynamics, vol. 84, no. 2, pp. 943-958, 2016.

[18] C. Yang, Q. Zhang, and L. Zhou, "Strongly absolute stability problem of descriptor systems: circle criterion," Journal of the Franklin Institute, vol. 345, no. 5, pp. 437-451, 2008.

[19] H. K. Khalil and J. W. Grizzle, Nonlinear Systems, PrenticeHall, Upper Saddle River, NJ, USA, 2002.

[20] G. Tao and P. A. Ioannou, "Necessary and sufficient conditions for strictly positive real matrices," IEE Proceedings G Circuits, Devices and Systems, vol. 137, no. 5, pp. 360-366, 1990.

[21] R. W. Freund and F. Jarre, "An extension of the positive real lemma to descriptor systems," Optimization Methods and Software, vol. 19, no. 1, pp. 69-87, 2004.

[22] M. Corless and R. Shorten, "On the characterization of strict positive realness for general matrix transfer functions," IEEE Transactions on Automatic Control, vol. 55, no. 8, pp. 18991904, 2010.

[23] P. L. Walsh and J. S. Lamancusa, "A variable stiffness vibration absorber for minimization of transient vibrations," Journal of Sound and Vibration, vol. 158, no. 2, pp. 195-211, 1992.

[24] T.-H. Wu and C.-C. Lan, "A wide-range variable stiffness mechanism for semi-active vibration systems," Journal of Sound and Vibration, vol. 363, pp. 18-32, 2016.

[25] F. Ferraguti, C. Secchi, and C. Fantuzzi, "A tank-based approach to impedance control with variable stiffness," in Proceedings of the 2013 IEEE International Conference on Robotics and Automation, pp. 4948-4953, Karlsruhe, Germany, May 2013. 